\title{
Isotherms and Streamlines for 2D Lid Driven Square Cavity
}

\author{
Garepally Srinivas, A.V Ramana Kumari, V. Narayana
}

\begin{abstract}
Analysis of lid driven square cavity flow of air with three different ranges of $R i$ and $R e$ are analyzed using numerically. Adiabatic temperature is maintained at horizontal walls and isothermal temperature is established at the vertical walls in which the top wall is assumed to slide with a uniform speed. Finite volume method techniques have used to solve non dimensional governing equations. To visualize the flow and thermal characteristics, the control parameters, the Richardson number (Ri) and Reynolds number (Re) and in the range of 0.001 $\leq R i \leq 10$ and $100 \leq R e \leq 400$ are used for streamlines and isotherms.
\end{abstract}

Keywords: Reynolds number, Richardson number, Mixed Convection.

\section{INTRODUCTION}

Much attention have been paid for the few decades on heat transfer flows with natural and mixed convective flows production, crystal growth etc. In different cavity configurations many authors [1-2] have considered the flow structure and its temperature distribution.

Moallemi and Jang [3] investigated the buoyancy effect in a $2 \mathrm{D}$ cavity with respect to $\operatorname{Pr}(0.01 \leq \operatorname{Pr} \leq 50), \operatorname{Re}\left(10^{2} \leq \operatorname{Re} \leq\right.$ 2000)and constant Grashof number (Gr). They observed that natural convection usually assists the forced convection. With top moving wall, similar cavity problem for different aspect ratios (AR) was analyzed by Prasad et al. [4] numerically in two different ways, namely, the top moving wall is maintained at a higher temperature in comparison with the bottom wall and vice versa. They noticed that when $\mathrm{AR}=$ 0.5 and 1.0 and for negatively increasing values of Gr, convection is strong inside the cavity. When AR increased to 2 and for $\mathrm{Gr}=-10^{5}$, Hopf bifurcation showed its presence. When AR value further increased to 10, Sharif [5] showed that along the heated sliding wall of the cavity the Nu shoots up and it decreases rapidly towards the right side of the wall.

In the present paper the contours of streamlines and

Manuscript received on September 15, 2021.

Revised Manuscript received on September 22, 2021.

Manuscript published on September 30, 2021.

* Correspondence Author

Garepally Srinivas*, Department of Mathematics, Balaji Institute of Technology \& Science, Warangal, Telangana, India Email: sreenugarepally@gmail.com

A. V. Ramana Kumari, Department of Mathematics, Kamala Institute of Technology \& Science, Singapuram, Karimnagar, Telangana, India Email: avrk.arvind@gmail.com

Narayana Vekamulla, Department of Mathematics, Balaji Institute of Technology \& Science, Warangal, Telangana, India Email: vnarayana.wgl@gmail.com

(c) The Authors. Published by Blue Eyes Intelligence Engineering and Sciences Publication (BEIESP). This is an open access article under the CC BY-NC-ND license (http://creativecommons.org/licenses/by-nc-nd/4.0/) which has many applications in food processing, glass

isotherms are used to visualize the behaviour of the flow.

\section{PHYSICAL MODEL AND GOVERNING EQUATIONS}

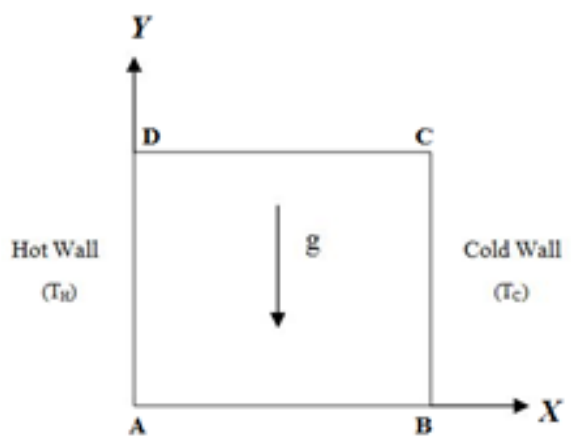

Fig. 1 2D Lid Driven Cavity

Figure 1 represents two dimensional lid driven cavity with velocity $U$ of length $L$. Air is the working fluid in which isothermal temperature is maintained at left and right walls and adiabatic temperature is maintained at top and bottom walls with $\left(T_{H}-T_{C}>0\right.$, and the boundary conditions are

$$
\begin{aligned}
& \Delta . \boldsymbol{V}=0 \\
& (\boldsymbol{V} . \nabla) \boldsymbol{V}=\frac{1}{R e} \nabla^{2} \boldsymbol{V}-\nabla p+R i T^{*} \boldsymbol{e} \\
& (\boldsymbol{V} . \nabla) T^{*}=\frac{1}{\operatorname{Pr} \cdot R e} \nabla^{2} T^{*}
\end{aligned}
$$

The boundary conditions are as follows

$\boldsymbol{V}=(1,0)$ at $Y=1$ and $\boldsymbol{V}=0$ at $Y=0, X=0,1$.

$T^{*}=0$ at $\mathrm{X}=1$ and $T^{*}=1$ at $X=0$ and

$\frac{\partial T^{*}}{\partial Y}=0$, at $Y=0$ and 1 .

\section{NUMERICAL METHOD}

The open source software Open FOAM has been used. For solving derivatives, the Second order upwind differencing scheme was employed to solve the spatial derivates. Divergent terms are discretized by QUICK scheme. Gauss linear method is used to solve Laplacian terms.

\section{RESULTS AND DISCUSSION}

Figure 2 displays the streamlines for different Ri and Re. It is found that the primary vortex take part a major role when $\mathrm{Ri}$ is small, with the increasing values of $\mathrm{Re}$ and it is changing from top position to bottom also it is found two tiny eddies at the bottom corners of the square cavity and also the size of tiny eddies are increases as Ri increases (Figs. 2(a, d)).

The primary major vortex moves towards the boundary walls and giving space for creating another vortex when $\mathrm{Ri}$ $=1$ and with increase of Re.

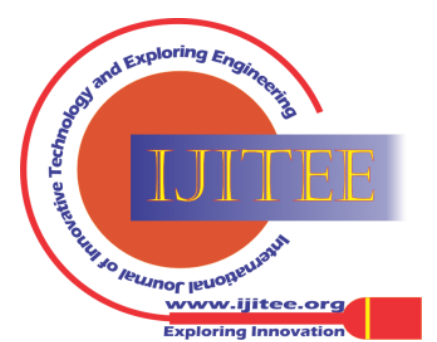




\section{Isotherms and Streamlines for 2D Lid Driven Square Cavity}

Finally two primary vortices formed when $\mathrm{Ri}=10$ and $\mathrm{Re}$ increases (Fig. 2(c)). The velocity of the fluid at the centre of the cavity are very small when compared with the velocities at the boundary when Re further increases.

(a)

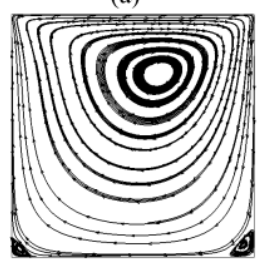

(D)

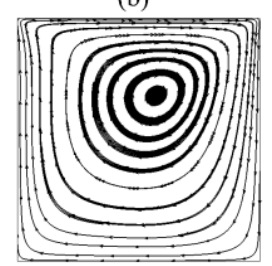

(c)

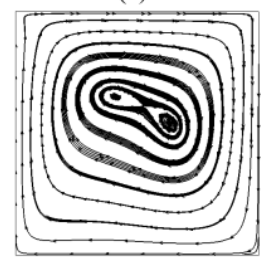

(d)

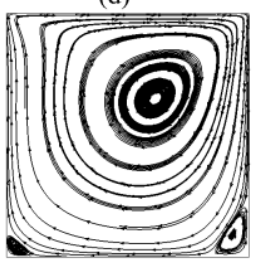

(e)

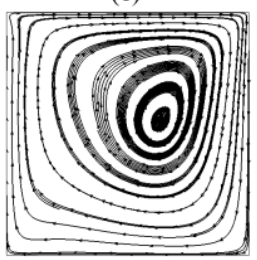

(f)

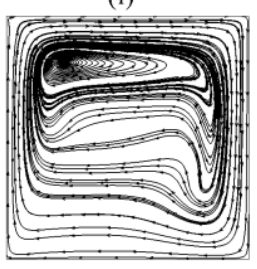

Fig. 2. Streamlines for different $\mathrm{Ri}$ and Re values. $(\mathrm{Ri}, \mathrm{Re})=(\mathrm{a})(0.001,100),(\mathrm{b})(1,100),(\mathrm{c})(10,100)$, (d) $(0.001,400),(e)(1,400),(f)(10,400)$

Figure 3 gives the isotherms for different values of $R i$ and $R e$. From the figure, it is noticed that vertical isotherms have formed near the boundaries when $R i$ is small, with the increase of $R e$, this is due to the conduction. As $R e$ further increases, due to the mechanism from the conduction mode to convection mode, the isotherm leaves from the vertical wall and found horizontal isotherms. Finally when $R i$ is further increases with the increase of $R e$, horizontal isotherms are observed in Figs. 3(c, f).

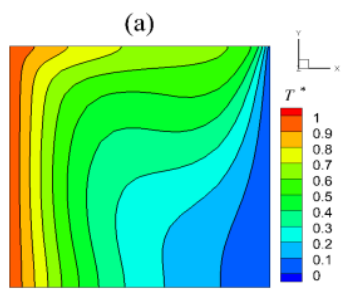

(b)

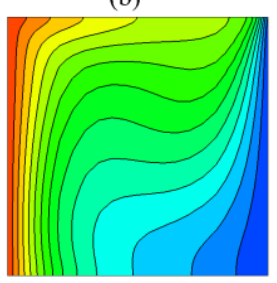

(c)

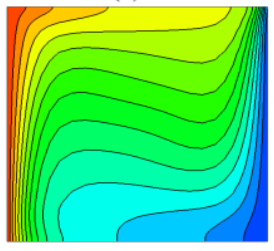

(d)

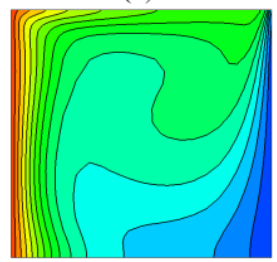

(e)

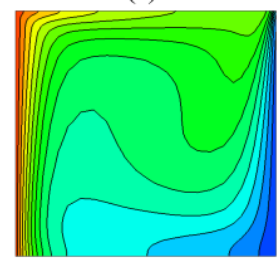

(f)

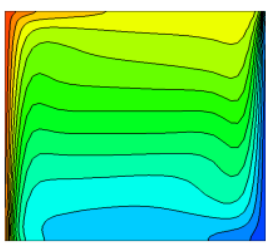

Fig. 3. Isotherms for different $\mathrm{Ri}$ and $\mathrm{Re}$ values. $(\mathrm{Ri}, \mathrm{Re})=(\mathrm{a})(0.001,100),(\mathrm{b})(1,100),(\mathrm{c})(10,100)$, (d) $(0.001,400),(e)(1,400),(f)(10,400)$

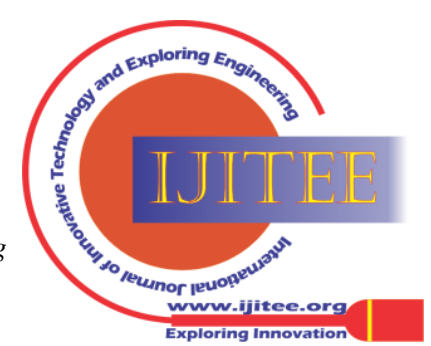




\section{CONCLUSION}

The present study is carried numerically for the mixed convective flow in a left heated lid driven 2D cavity for different ranges of $R i$ and $R e$. The simulated results of streamlines exhibited symmetric nature at the horizontal adiabatic walls. When $R i$ is small with the increase of $R e$, the primary vortex inhabited the major part of the cavity and also horizontal isotherms formed near the boundaries due to conduction.

\section{REFERENCES}

1. deVahl Davis, G and Jones, I.P (1983). Natural convection in a square cavity: a comparison exercise, International Journal of Numerical Methods in Fluids, Vol. 3, 227-248.

2. Markatos, N.C and Pericleous, K.A (1984). Laminar and turbulent natural convection in an enclosed cavity, International Journal of Heat and Mass Transfer, Vol. 27, 755-772.

3. Moallemi M.K and Jang, K.S (1992). Prandtl number effects on laminar mixed convection heat transfer in a lid-driven cavity, International Journal of Heat and Mass Transfer, Vol. 35, 1881-1892.

4. Prasad, Y.S and Das, M.K (2007). Hopf bifurcation in mixed flow inside a rectangular cavity, International Journal of Heat and Mass Transfer, Vol. 50, 3583-3598.

5. Sharif, M.A.R (2007). Laminar mixed convection in shallow inclined driven cavities with hot moving lid on top and cooled from bottom, Applied Thermal Engineering, Vol. 27 1036-1042.

6. V.Narayana, H.P. Rani. (2020) Analysis of visualization techniques of bottom heated lid driven square cavity, Heat Transfer, Vol. 49, issue 6, pp. 1-11

7. V.Narayana. (2020) Analysis of new visualization techniques of left heated lid driven square cavity, Heat Transfer, Vol. 49, issue 3, pp. $1-9$.

\section{AUTHORS PROFILE}

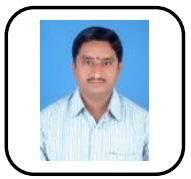

Mr. Garepally Srinivas has been working in Balaji Institute of Technology \& Science since 2017. He has completed his M.Sc Applied Mathematics at NIT Warangal and presently pursuing $\mathrm{Ph}$.D from JNTU Hyderabad. He has 24 years of Teaching experience. He has attended 10 international conferences and presented 5 papers. Also he has organized 3 National Level FDP and Workshops.

Dr. A. V. Ramana Kumari has been working in Kamala Institute of Technology \& Science, since 1997. She has completed her M.Sc Applied Mathematics and Ph.D from NIT Warangal. She has 24 years of Experience. She has 10 publications and attended 10 International and National conferences and organized 5 workshops and conferences.

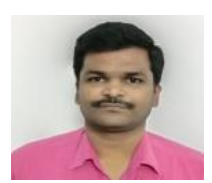

Dr.V. Narayana, has been working in Balaji Institute of Technology and Science since 2007. He completed his M.Sc.(Mathematics) and Ph.D. in from National Institute of Technology, Warangal. He is presently working as a Academic Coordinator. He has 14 years of teaching experience. He has published 14 research papers in Scopus Indexed Journals.

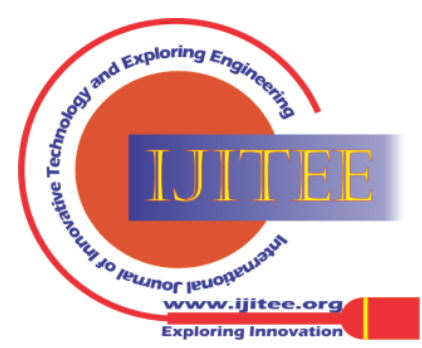

